Draft version, 12/01/18. This paper has not been peer reviewed. Please do not copy or cite without authors' permission. This notice is posted in compliance with APA Journals' Internet Posting Guidelines.

\title{
Proactive versus reactive emotion regulation: A dual-mechanisms perspective
}

\author{
Bruna Martins ${ }^{1}$, Lyneé A. Alves², Kimberly S. Chiew²
}

${ }^{1}$ Department of Psychological and Brain Sciences, University of Massachusetts-

Amherst

${ }^{2}$ Department of Psychology, University of Denver

Corresponding Author:

Kimberly S. Chiew, Ph.D.

Department of Psychology

University of Denver

2155 S. Race Street

Denver, CO USA 80208

kimberly.chiew@du.edu 


\begin{abstract}
Emotion regulation relies on cognitive processing, but the foundational cognitive control mechanisms engaged remain unclear. The process model of emotion regulation posits that different strategies occur at different points in time, with antecedent strategies occurring early and response-focused strategies later in the affective timecourse, with cognitive processing supporting these strategies following a similar timecourse. In parallel, the Dual Mechanisms of Control (DMC) theoretical framework (Braver, 2012; Braver, Gray, \& Burgess, 2007; Chiew \& Braver, 2017) proposes that cognitive control operates via two temporally-distinct modes: anticipatory preparation to perform cognitive tasks (proactive), and momentary engagement in cognitive tasks as they arise (reactive). However, empirical investigations of the role of proactive and reactive control in emotion regulation have been limited. In this paper, we summarize and integrate these two theoretical perspectives. We first posit that any emotion regulation strategy may take place either early or late in the affective timecourse, depending on whether it is proactively or reactively enacted. We next provide examples of different strategies from the process model and their engagement in both control modes. In addition, we discuss how strategic dependence on the downstream emotional stimulus and response could further affect the timecourse and cognitive load of emotion regulation strategies. We conclude by discussing how controlling for timing in future research designs may clarify how populations with reduced cognitive control may demonstrate intact emotion regulation (i.e., through greater reliance on reactive strategies), and how incorporation of the DMC perspective may inform applied emotion regulation interventions for clinical populations.
\end{abstract}


Keywords: emotion regulation, cognitive control, temporal dynamics, dual mechanisms 
As we navigate the richness and complexity of daily life, our emotions are critical both as drivers and outcomes of behavior. Emotional responses can be highly adaptive and crucial to survival, motivating reward approach as well as avoidance of environmental dangers (Panksepp, 2004). Mechanisms that facilitate adaptive control and minimize maladaptive control of emotions, or emotion regulation (ER), are important - emotion dysregulation is indicative of poor psychological outcomes, including depression, anxiety, and substance abuse (Aldao \& Nolen-Hoeksema, 2012). While the link between cognitive control and emotion regulation is widely accepted in the field, the specific control processes involved in ER remain under-characterized. Within the cognitive literature, the Dual Mechanisms of Control (DMC) framework (Braver, 2012; Braver et al., 2007; Chiew \& Braver, 2017) has been an influential conceptualization of cognitive control as operating in two modes: one mode characterized by anticipatory preparation to use control (proactive), and the other characterized by use of in-the-moment, flexible control engagement as the need for control arises (reactive). This framework has been used to formally characterize the cognitive processes, brain mechanisms, and physiological timecourses by which adaptive cognitive control operates. However, potential distinctions between proactive and reactive ER have been underexplored to date. In the present paper, we review the DMC framework as a theoretical model for understanding cognitive control processes, and argue for the utility of explicitly characterizing ER processes in terms of this framework. We apply the DMC framework to ER by: 1) reviewing the classic DMC model and proactive versus reactive models of cognitive control; 2) discussing how proactive versus reactive ER may operate via differing temporal dynamics; 3) arguing 
for a model of dual mechanisms of control in ER, which conceptualizes the role of cognitive control in ER in terms of regulation choice onset, and reliance of specific strategies on situational context (e.g., reliance on emotional stimulus and/or response). Finally, we close by discussing the impact of this model on understanding ER in populations with cognitive control difficulties, and overviewing future directions for research generated by this model and its synthesis.

\section{The DMC model and cognitive control across proactive and reactive modes}

The DMC framework posits two separate but complementary methods for orchestrating cognitive control in support of adaptive goal-directed behavior. The first mode, proactive control, is characterized by sustained or preparatory goal maintenance, engaged prior to anticipated task demands. In contrast, reactive control may act as a 'late correction' mechanism, solicited in response to transient environmental signals such as feedback or conflict. While no "hard and fast" distinction between proactive and reactive control modes exists, proactive control is posited to be relatively more sustained and/or emerging earlier, in a preparatory manner, while reactive control is posited to be relatively transient and emerge later, in response to control demands as they arise. Further, theoretical accounts of DMC have linked proactive control specifically to active maintenance of information in working memory and sustained or anticipatory activity in dorsolateral prefrontal cortex (DLPFC; Braver, 2012). Much of the empirical evidence supporting this characterization has utilized the AX Continuous Performance Task (AX-CPT), a cue-probe-response task where participants must actively maintain cue (context) information as well as flexibly respond to probe targets; 
the majority of trial responses are predicted in advance by the cue, and thus performance benefits from cue maintenance and advance preparation. Early fMRI studies of the AX-CPT (Barch et al., 1997; Braver \& Cohen, 2001) suggest that active cue maintenance over extended delays (5-10 seconds) is associated with more robust DLPFC activity than maintenance over shorter delays (1-2 seconds).

Critically, previous investigations have used paradigms with clear distinctions between cue and probe processing stages and analyses of neural activity at each stage to disentangle potential neural substrates supporting proactive versus reactive cognitive control. From a practical viewpoint, these observations may present important design considerations for future ER studies. Specifically, while many ER studies use anticipatory cues to precede emotional probes, effects of cue-probe delay manipulations are rarely systematically investigated, and cue and probe processing stages are not necessarily modeled separately in neuroimaging data (Kalisch, 2009).

\section{Emotion regulation has established link with cognitive control processes}

Extensive empirical evidence links ER mechanisms to fundamental cognitive control processes. ER strategies such as positive reappraisal (seeing the 'silver lining' in a negative situation) may rely on a number of controlled cognitive processes, including: keeping the initial interpretation of a situation in mind; generating alternate interpretations of that situation; and updating the meaning of the negative situation to the reappraisal that is most helpful (Ochsner, Silvers, \& Buhle, 2012). Reappraisal success has been linked to core cognitive processes including working memory capacity and set-shifting ability (McRae, Jacobs, Ray, John, \& Gross, 2012) as well as 
fluid reasoning (Opitz, Lee, Gross, \& Urry, 2014). In addition to reappraisal, working memory performance has been associated with other ER strategies, including capacity to inhibit emotional reactions to negative films (Schmeichel, Volokhov, \& Demaree, 2008), spontaneous self-soothing following interpersonal criticism (Schmeichel \& Demaree, 2010) and successful regulation of reactions to daily life stressors (Stawski, Almeida, Lachman, Tun, \& Rosnick, 2010). Successful ER has also been linked to increased activity in prefrontal and parietal cortex regions associated with cognitive control (Dosenbach et al., 2007; Morawetz, Bode, Derntl, \& Heekeren, 2017) and decreased activity in regions implicated in emotion and salience processing, such as the amygdala (Ochsner, Bunge, Gross, \& Gabrieli, 2002). In addition to changes in brain activity, the functional connectivity between prefrontal regions and the amygdala has been shown to increase with ER success (Urry et al., 2006; Winecoff, LaBar, Madden, Cabeza, \& Huettel, 2010). However, despite extensive literature suggesting that working memory and cognitive control abilities are crucial to ER processing, implementation of proactive versus reactive control processes in ER has yet to be systematically disentangled.

\section{Proactive and reactive emotion regulation may differ in their temporal dynamics}

A critical element of the DMC framework's proactive and reactive control modes is their differing temporal dynamics. While ER has yet to be fully characterized in terms of the DMC framework, the temporal dimension of affective processing and its regulation has been recognized in the process model of emotion regulation (Gross, 2014). This model posits that emotional experience and response unfold in sequential 
stages, starting with exposure to an emotional stimulus and culminating in outward emotional expression, with multiple stages in between where regulatory processes can potentially intervene and modify the developing emotional response (Gross, 2014). Within the process model, researchers have distinguished between early-onset antecedent regulatory strategies, such as situation selection, attentional deployment, reappraisal, and distraction; these are conceptualized as occurring prior to an emotional response, relatively early in the affective timecourse. In contrast, response-focused strategies, such as the suppression of emotional expression, take place relatively late in the timecourse and alter an emotional response once underway (Dan-Glauser \& Gross, 2011). Antecedent versus response-focused strategies and their differing effects in the affective timecourse have been characterized in terms of neural activity using both EEG (Moser, Krompinger, Dietz, \& Simons, 2009; Thiruchselvam, Blechert, Sheppes, Rydstrom, \& Gross, 2011) and fMRI (Waugh et al., 2016). Timing is also important within strategy type: over time, reappraisal processing engages early left posterior and later right anterior portions of the lateral PFC (Kalisch, 2009; Paret et al., 2011). While theoretical models argue that this pattern of PFC involvement may reflect early implementation in working memory and later maintenance of ER strategies, only a few investigations of ER task timing have attempted to characterize these effects (Denny, Ochsner, Weber, \& Wager, 2013; Vanderhasselt, Kühn, \& De Raedt, 2012).

\section{Use of dual control mechanisms in emotion regulation is modulated by regulation onset and dependence on stimulus or response}


Within the broader consideration of temporal dynamics in ER, strategy timing has been identified as a potential contributor to ER success (Sheppes \& Gross, 2011). Despite this, most investigations of timing effects in ER still conceptualize specific strategies as occurring at fixed, specific points in the affective timecourse, without accounting for the fact that most regulation strategies can be implemented in a proactive or reactive manner. We thus suggest that, rather than associating a given regulation strategy with a specific phase in the affective timecourse, different ER strategies may be implemented in either a proactive or a reactive manner, depending on when the regulation choice is made. This decision may occur either in advance of or in reaction to an emotionally evocative stimulus or response. Thus, any given strategy may potentially be used at a relatively early or late phase within the timecourse, depending on when one starts preparing to use that strategy. In Figure 1, we propose theoretical timecourses for different strategies from the process model of ER, and propose that the onset of regulatory control may vary based on whether strategies are utilized in a proactive versus reactive mode.

In addition to varying in terms of timing, we also argue that ER strategies may vary in terms of dependency on the emotionally evocative stimulus or elicited response (thus, ER strategies can be considered stimulus-dependent or stimulus-independent, response-dependent or response-independent). For instance, with situation selection as a strategy, one could globally choose to avoid all emotional situations (proactive), or choose to leave an emotional situation once it begins (reactive). With attentional deployment, one could globally divert attention away from upcoming emotional stimuli (proactive stimulus-independent) or redirect attention specifically away from emotional 
portions of a specific stimulus upon presentation (reactive stimulus-dependent). Interestingly, even if a strategy is stimulus- or response-dependent, one could use it in a deliberative or anticipatory manner. For instance, one could prepare to redirect attention away from emotional portions of upcoming negative stimuli (proactive stimulusdependent). With positive reappraisal, one could use a global, one-size-fits-all reappraisal (e.g., "it will be okay in the end") when interpreting an anticipated negative emotional situation (proactive) or reappraise the meaning of a specific emotional stimulus (e.g, interpret it as less negative) as it is encountered (reactive). Finally, suppression of an emotional response could involve muting emotional expressivity towards an anticipated emotional situation (e.g., proactively "steeling oneself" before entering the situation) or inhibiting an outward emotional expression after the experience and response elicited by an emotional stimulus has begun (reactive). This conceptualization of ER strategies as proactive or reactive, stimulus and response dependent or independent, represents an expansion of the process model by positing that a given ER strategy may be flexibly implemented in a proactive or reactive mode at different phases of the affective timecourse, rather than being associated with a specific timepoint in the process.

Proposed timecourses of ER strategies as potentially proactive or reactive, stimulus dependent or independent, and response dependent or independent are presented in Figure 1. Additionally, Table 1 highlights specific experimental paradigms that can be understood as consistent with these categorizations. Importantly, these categorizations are post-hoc; ER strategies and their neural substrates will need to be investigated in experimental paradigms deliberately designed to draw on proactive 
versus reactive control mechanisms, to confirm the utility and validity of these distinctions.

[Figure 1 and Table 1 somewhere around here]

\section{Utility and applications of a DMC framework for advancing understanding of emotion regulation}

As discussed above, ER strategies can be understood as operating in either a proactive or reactive fashion, but most ER investigations have not been designed to separate these control modes from one another. We suggest that characterizing ER in terms of this theoretical framework will help account for performance variability by advancing our understanding of the control mechanisms involved, their neural substrates, and the timing by which they are engaged. This parallels the utility of the DMC framework in the study of classic cognitive control, where it has been used to account for variability in and formulate testable hypotheses regarding cognitive performance across situational contexts, across individuals, and across population groups (Braver, 2012). Multiple factors can contribute to ER success; while it is clear that cognitive control is involved in ER, the DMC framework sets up formal predictions for the contributions of different control modes in a mechanistically-explicit way.

Specifically, the DMC approach may help elucidate longstanding questions regarding variability in ER performance across populations. For example, a "paradox of aging" has been suggested: cognitive control decline in older adults $(O A)$ is welldocumented, yet superior ER and well-being has been characterized in this age group 
relative to younger adults (Mather, 2012). Given the importance of cognitive control processes to ER, this paradox has posed a riddle to researchers. Studies investigating basic cognitive control in OA using a dual-mechanisms perspective suggest that older adults have a specific deficit in proactive control, while reactive control remains relatively preserved (Paxton, Barch, Storandt, \& Braver, 2006; Paxton, Barch, Racine, \& Braver, 2007). Given the preservation of reactive control in this population, the superior ER abilities observed in older adults may be linked to preferential use of strategies depending on less cognitive control, and more reactive rather than proactive control. More cognitively demanding strategies such as reappraisal are more effortful than simple distraction for older adults (Martins, Florjanczyk, Jackson, Gatz, \& Mather, 2018), but the contributions of proactive versus reactive control mechanisms across different age groups in ER has yet to be clarified. While the cognitive literature often depicts reactive control as associated with poorer task performance (Edwards, Barch, \& Braver, 2010; Paxton et al., 2007), the potential adaptive value of reactive strategies remains unknown.

Clarifying the effort involved in proactive and reactive control, and how it impacts the utility of ER strategy use, is also crucial to advancing therapeutic interventions. Further, clarifying differences in the efficacy of stimulus-focused and response-focused versus more global strategies will be helpful in tailoring interventions to specific groups. For instance, older adults with late-life mood disorders may demonstrate different costs and benefits of proactive preparation to regulate (engaging in daily morning mindfulness) versus reactive regulation (engaging in a grounding strategy following a distressing situation). Other populations with ER and cognitive control difficulties, such 
as children with conduct problems and patients with schizophrenia, may also

emotionally benefit from this future research. Proactive control training has been shown to improve cognitive performance in patients with schizophrenia (Edwards et al., 2010); it is unclear whether proactive ER training may similarly benefit downstream emotion processing. If proactive control of emotion leads to greater emotional benefits, cognitive training (perhaps implemented via mobile reminders, wearables and apps) could help boost use of anticipatory strategies in populations that struggle to engage these strategies independently.

\section{Conclusion}

The DMC framework has important utility in clarifying the control mechanisms underlying ER and variability in its performance in a range of situations and at multiple levels of analysis. As reviewed, most experimental paradigms investigating ER do not explicitly characterize contributions of these two control modes, nor do they differentiate stimulus- and response-specific strategies from those that are more global. We thus suggest that an important direction for future ER research is to utilize experimental designs that deliberately engage regulation processes in a proactive versus reactive fashion and enable characterization of the temporal dynamics of these processes as they unfold.

Considering the DMC perspective will enable further application of theoreticallymotivated predictions from the classic cognitive control literature, strengthening existing models of emotion regulation. This integrative approach may help clarify some of the 
longstanding puzzles regarding the mechanisms that support successful emotion regulation and their variability across populations.

\section{$\underline{\text { References }}$}

Aldao, A., \& Nolen-Hoeksema, S. (2012). When are adaptive strategies most predictive of psychopathology? Journal of Abnormal Psychology, 121(1), 276.

Barch, D. M., Braver, T. S., Nystrom, L. E., Forman, S. D., Noll, D. C., \& Cohen, J. D. (1997). Dissociating working memory from task difficulty in human prefrontal cortex. Neuropsychologia, 35(10), 1373-1380. Retrieved from http://www.ncbi.nlm.nih.gov/entrez/query.fcgi?cmd=Retrieve\&db=PubMed\&dopt=Ci tation\&list_uids $=9347483$

Braver, T. S. (2012). The variable nature of cognitive control: a dual mechanisms framework. Trends Cogn Sci, 16(2), 106-113. https://doi.org/10.1016/j.tics.2011.12.010

Braver, T. S., \& Cohen, J. D. (2001). Working memory, cognitive control, and the prefrontal cortex: Computational and empirical studies. Cognitive Processing, 2(1), $25-55$.

Braver, T. S., Gray, J. R., \& Burgess, G. C. (2007). Explaining the many varieties of working memory variation: dual mechanisms of cognitive control. In A. R. A. Conway, C. Jarrold, M. J. Kane, \& A. Miyake Towse, J.N. (Eds.), Variation in working memory (pp. 76-106). Oxford: Oxford University Press.

Chiew, K. S., \& Braver, T. S. (2017). Context processing and cognitive control: From gating models to dual mechanisms. The Wiley Handbook of Cognitive Control, 
$143-166$.

Dan-Glauser, E. S., \& Gross, J. J. (2011). The temporal dynamics of two responsefocused forms of emotion regulation: Experiential, expressive, and autonomic consequences. Psychophysiology, 48(9), 1309-1322.

Denny, B. T., Ochsner, K. N., Weber, J., \& Wager, T. D. (2013). Anticipatory brain activity predicts the success or failure of subsequent emotion regulation. Social Cognitive and Affective Neuroscience, 9(4), 403-411.

Dosenbach, N. U. F., Fair, D. A., Miezin, F. M., Cohen, A. L., Wenger, K. K., Dosenbach, R. A. T., ... Raichle, M. E. (2007). Distinct brain networks for adaptive and stable task control in humans. Proceedings of the National Academy of Sciences, 104(26), 11073-11078.

Edwards, B. G., Barch, D. M., \& Braver, T. S. (2010). Improving prefrontal cortex function in schizophrenia through focused training of cognitive control. Front Hum Neurosci, 4, 32. Retrieved from http://www.ncbi.nlm.nih.gov/entrez/query.fcgi?cmd=Retrieve\&db=PubMed\&dopt=Ci tation\&list_uids $=20461148$

Gross, J. J. (2014). Emotion regulation: Conceptual and empirical foundations.

Kalisch, R. (2009). The functional neuroanatomy of reappraisal: time matters. Neuroscience \& Biobehavioral Reviews, 33(8), 1215-1226.

Martins, B., Florjanczyk, J., Jackson, N. J., Gatz, M., \& Mather, M. (2018). Age differences in emotion regulation effort: Pupil response distinguishes reappraisal and distraction for older but not younger adults. Psychology and Aging, 33(2), 338. Mather, M. (2012). The emotion paradox in the aging brain. Annals of the New York 
Academy of Sciences, 1251(1), 33-49.

McRae, K., Jacobs, S. E., Ray, R. D., John, O. P., \& Gross, J. J. (2012). Individual differences in reappraisal ability: Links to reappraisal frequency, well-being, and cognitive control. Journal of Research in Personality, 46(1), 2-7.

Morawetz, C., Bode, S., Derntl, B., \& Heekeren, H. R. (2017). The effect of strategies, goals and stimulus material on the neural mechanisms of emotion regulation: A meta-analysis of fMRI studies. Neuroscience \& Biobehavioral Reviews, 72, 111128.

Moser, J. S., Krompinger, J. W., Dietz, J., \& Simons, R. F. (2009). Electrophysiological correlates of decreasing and increasing emotional responses to unpleasant pictures. Psychophysiology, 46(1), 17-27.

Ochsner, K. N., Bunge, S. A., Gross, J. J., \& Gabrieli, J. D. (2002). Rethinking feelings: an FMRI study of the cognitive regulation of emotion. J Cogn Neurosci, 14(8), 1215-1229. Retrieved from http://www.ncbi.nlm.nih.gov/entrez/query.fcgi?cmd=Retrieve\&db=PubMed\&dopt=Ci tation\&list_uids $=12495527$

Ochsner, K. N., Silvers, J. A., \& Buhle, J. T. (2012). Functional imaging studies of emotion regulation: a synthetic review and evolving model of the cognitive control of emotion. Ann N Y Acad Sci, 1251, E1-24. https://doi.org/10.1111/j.1749$6632.2012 .06751 . x$

Opitz, P. C., Lee, I. A., Gross, J. J., \& Urry, H. L. (2014). Fluid cognitive ability is a resource for successful emotion regulation in older and younger adults. Frontiers in Psychology, 5, 609. 
Panksepp, J. (2004). Affective neuroscience: The foundations of human and animal emotions. Oxford university press.

Paret, C., Brenninkmeyer, J., Meyer, B., Yuen, K. S., Gartmann, N., Mechias, M.-L., \& Kalisch, R. (2011). A test for the implementation-maintenance model of reappraisal. Frontiers in Psychology, 2, 216.

Paxton, J. L., Barch, D. M., Racine, C. A., \& Braver, T. S. (2007). Cognitive control, goal maintenance, and prefrontal function in healthy aging. Cerebral Cortex, 18(5), 1010-1028.

Paxton, J. L., Barch, D. M., Storandt, M., \& Braver, T. S. (2006). Effects of environmental support and strategy training on older adults' use of context. Psychol Aging, 21(3), 499-509. https://doi.org/10.1037/0882-7974.21.3.499

Schmeichel, B. J., \& Demaree, H. A. (2010). Working memory capacity and spontaneous emotion regulation: High capacity predicts self-enhancement in response to negative feedback. Emotion, 10(5), 739.

Schmeichel, B. J., Volokhov, R. N., \& Demaree, H. A. (2008). Working memory capacity and the self-regulation of emotional expression and experience. Journal of Personality and Social Psychology, 95(6), 1526.

Sheppes, G., \& Gross, J. J. (2011). Is timing everything? Temporal considerations in emotion regulation. Personality and Social Psychology Review, 15(4), 319-331.

Stawski, R. S., Almeida, D. M., Lachman, M. E., Tun, P. A., \& Rosnick, C. B. (2010). Fluid cognitive ability is associated with greater exposure and smaller reactions to daily stressors. Psychology and Aging, 25(2), 330.

Thiruchselvam, R., Blechert, J., Sheppes, G., Rydstrom, A., \& Gross, J. J. (2011). The 
temporal dynamics of emotion regulation: An EEG study of distraction and reappraisal. Biological Psychology, 87(1), 84-92.

Urry, H. L., Van Reekum, C. M., Johnstone, T., Kalin, N. H., Thurow, M. E., Schaefer, H. S., ... Alexander, A. L. (2006). Amygdala and ventromedial prefrontal cortex are inversely coupled during regulation of negative affect and predict the diurnal pattern of cortisol secretion among older adults. Journal of Neuroscience, 26(16), 44154425.

Vanderhasselt, M.-A., Kühn, S., \& De Raedt, R. (2012). 'Put on your poker face': neural systems supporting the anticipation for expressive suppression and cognitive reappraisal. Social Cognitive and Affective Neuroscience, 8(8), 903-910.

Waugh, C. E., Zarolia, P., Mauss, I. B., Lumian, D. S., Ford, B. Q., Davis, T. S., ... McRae, K. (2016). Emotion regulation changes the duration of the BOLD response to emotional stimuli. Social Cognitive and Affective Neuroscience, 11(10), 15501559.

Winecoff, A., LaBar, K. S., Madden, D. J., Cabeza, R., \& Huettel, S. A. (2010). Cognitive and neural contributors to emotion regulation in aging. Social Cognitive and Affective Neuroscience, 6(2), 165-176. 


\section{Figure \& Table Captions}

Figure 1. Proposed timecourses of ER strategies as potentially proactive or reactive, stimulus dependent or independent, and response dependent or independent, in combination with one another. As indicated in the different profiles of the timecourses, regulatory control processes can be implemented in a more phasic, event-locked fashion; a more global, sustained fashion; or a combination of the two. Further, control can be implemented at different stages in the affective process.

Table 1. Examples of specific experimental paradigms consistent with the proposed regulatory timecourses in Figure 1, with brief descriptions of experimental task, timecourse of emotion and its regulation, and key outcomes. Note that these categorizations are post-hoc; new paradigms deliberately designed to characterize proactive or reactive ER processes are called for. 


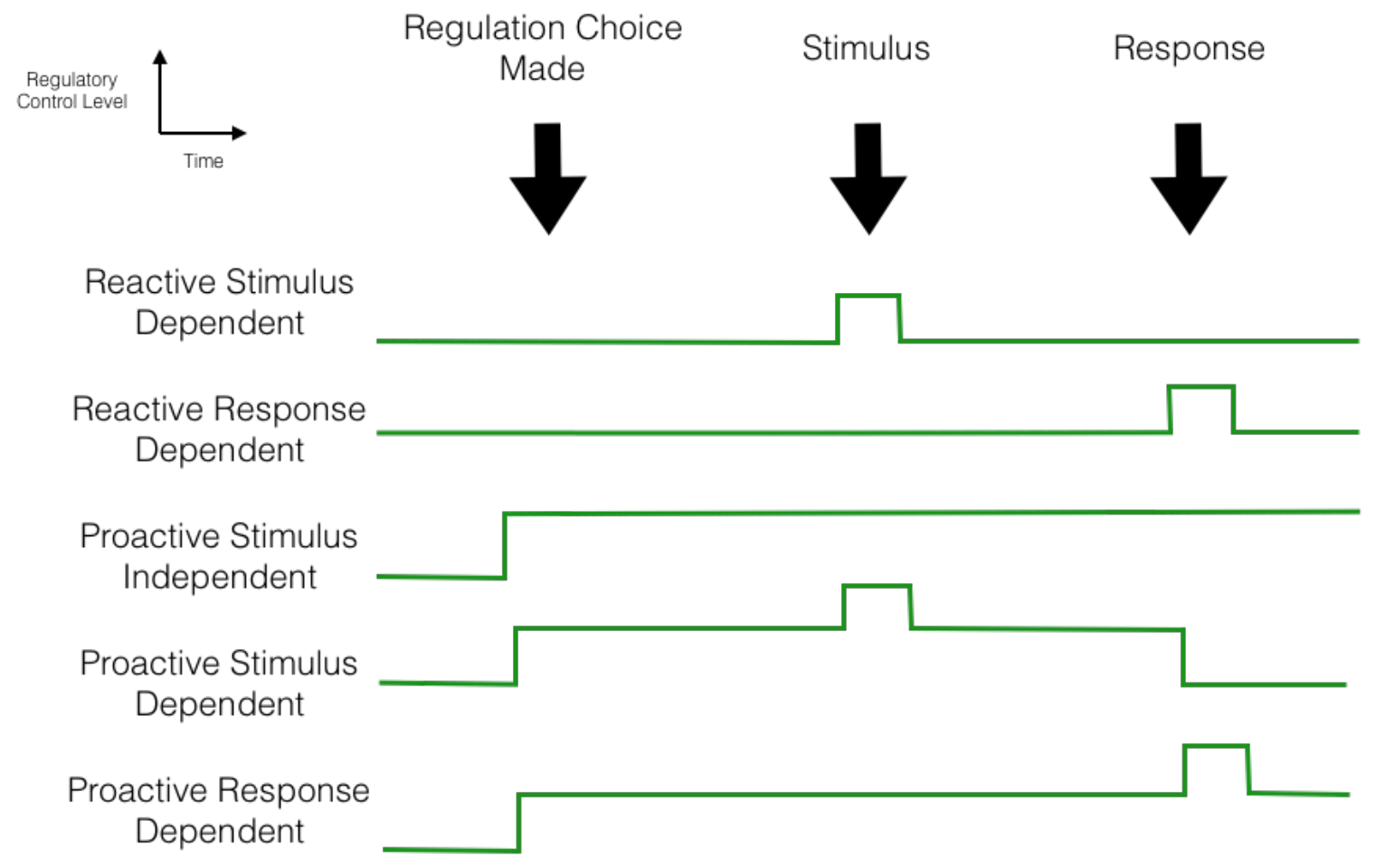

Figure 1. 


\begin{tabular}{|c|c|}
\hline \multicolumn{2}{|c|}{ Reactive, Stimulus-Dependent } \\
\hline \multicolumn{2}{|r|}{$\begin{array}{l}\text { Hajack \& Nieuwenhuis, } 2006 \\
\text { After viewing unpleasant images, participants either reinterpreted or simply attended to the image } \\
\text { - Greater reduction in LPP response vs. baseline, during reappraisal vs. passive viewing }\end{array}$} \\
\hline \multicolumn{2}{|c|}{ Reactive, Response Dependent } \\
\hline \multicolumn{2}{|r|}{$\begin{array}{l}\text { Chiew \& Braver, } 2010 \\
\text { - Participants cued to either smile or frown, then viewed a positive or negative image } \\
\text {. Participants responded with facial expressions to cue-image combinations } \\
\text {. Conflict trials required a facial expression opposite to image valence (e.g., smile after viewing a negative image) } \\
\text {. Responses were slower and more error-prone when image and expression valence conflicted }\end{array}$} \\
\hline \multicolumn{2}{|c|}{ Proactive, Stimulus-Dependent } \\
\hline \multicolumn{2}{|r|}{$\begin{array}{l}\text { Goldin et al., } 2007 \\
\text { - Participants were trained on both reappraisal and suppression } \\
\text {. } \text { Cued to engage in one strategy or the other before viewing disgust-inducing videos in an fMRI scanner } \\
\text {. Only reappraisal trials showed a decrease in activity in emotion brain regions }\end{array}$} \\
\hline \multicolumn{2}{|r|}{ Proactive, Stimulus-Independent } \\
\hline \multicolumn{2}{|r|}{$\begin{array}{l}\text { Herwig et al., } 2007 \\
\text { - Participants trained on reality checking, a form of reappraisal; a control group received no training } \\
\text {. Compared neural responses when participants were cued vs. uncued to the valence of the upcoming image } \\
\text { greater increase in activity for cognitive control regions during image viewing } \\
\text {. Trained participants showed greater decrease in emotional experience for both cued and uncued trials vs. untrained } \\
\text { participants }\end{array}$} \\
\hline \multicolumn{2}{|c|}{ Proactive, Response-Dependent } \\
\hline & $\begin{array}{l}\text { During EEG, participants were cued to passively view, up-regulate emotional responses to, or down-regulate } \\
\text { emotional responses to, an upcoming unpleasant or neutral image } \\
\text { LPP magnitude varied with the direction of emotion regulation: up-regulation of emotion increased the magnitude of } \\
\text { the LPP and down-regulation of emotion decreased the LPP }\end{array}$ \\
\hline
\end{tabular}

\section{Table 1.}

